\title{
Local Stability Analysis and Bifurcations of a Discrete-Time Host-Parasitoid Model
}

\author{
Tahmineh Azizi \\ Department of Mathematics, Kansas State University, Manhattan, KS, USA \\ Email: tazizi@ksu.edu
}

How to cite this paper: Azizi, T. (2020) Local Stability Analysis and Bifurcations of a Discrete-Time Host-Parasitoid Model. International Journal of Modern Nonlinear Theory and Application, 9, 19-33. https://doi.org/10.4236/ijmnta.2020.92002

Received: April 5, 2020

Accepted: June 7, 2020

Published: June 10, 2020

Copyright $\odot 2020$ by author(s) and Scientific Research Publishing Inc. This work is licensed under the Creative Commons Attribution International License (CC BY 4.0).

http://creativecommons.org/licenses/by/4.0/ (c) (i) Open Access

\begin{abstract}
In this paper, we examine a discrete-time Host-Parasitoid model which is a non-dimensionalized Nicholson and Bailey model. Phase portraits are drawn for different ranges of parameters and display the complicated dynamics of this system. We conduct the bifurcation analysis with respect to intrinsic growth rate $r$ and searching efficiency a. Many forms of complex dynamics such as chaos, periodic windows are observed. Transition route to chaos dynamics is established via period-doubling bifurcations. Conditions of occurrence of the period-doubling, Neimark-Sacker and saddle-node bifurcations are analyzed for $b \neq a$ where $a, b$ are searching efficiency. We study stable and unstable manifolds for different equilibrium points and coexistence of different attractors for this non-dimensionalize system. Without the parasitoid, the host population follows the dynamics of the Ricker model.
\end{abstract}

\section{Keywords}

Chaos, Neimark-Sacker Bifurcation, Period-Doubling Bifurcations, Manifold, Saddle-Node Bifurcation

\section{Introduction}

More efficient computational models for numerical simulation can be created by discrete-time models and also these models can present much more plentiful dynamic behaviors when they would be compared with the same type of continuous-time model [1] [2] [3]. In fact, when populations have non-overlapping generations, discrete-time models will be more rational and applicable than the continuous time models [1] [2]. In the 1935, Nicholson and Bailey could develop a discrete-time model for the population dynamics of insect hosts and their parasitoids [2] [4]. 
In this paper, we consider a particular case of the following general model [3] [4]:

$$
\left\{\begin{array}{l}
N_{t+1}=N_{t} \mathrm{e}^{\left[r\left(1-N_{t} / k\right)-b P_{t}\right]}, \\
P_{t+1}=N_{t}\left(1-\mathrm{e}^{-a P_{t}}\right),
\end{array}\right.
$$

where, $k$ is the carrying capacity and shows maximum population size that can be supported by availability of all the potentially limiting resources. It is usually limited by the intensity of light and space. Since $k$ is easily affected by the environment, it cannot be always a constant. The fractions of hosts not parasitized are $\mathrm{e}^{-a P_{t}}, \mathrm{e}^{-b P_{t}}$ where $a, b$ are searching efficiency (or the probability that $a, b$ given parasitoid face a host whole of their lifetime). $\mathrm{e}^{r\left(1-N_{t} / k\right)}$ is the density-dependent factor [2]. Density-dependent factor affects host populations. For some parameter values the density-dependent factor has a stabilizing impact. If the parameter $r$ is not high, there will exist a locally asymptotically stable equilibrium.

We investigate the stability for $b \neq a$. We do the bifurcation analysis with respect to intrinsic growth rate $r$ and searching efficiency $a$ for $b \neq a$. In the absence of the parasitoid, the dynamics of this non-dimensionalized model are the same as the dynamics which is given by the Ricker map. Finally, we represent the non-dimensionalized model:

$$
\left\{\begin{array}{l}
x_{t+1}=x_{t} \mathrm{e}^{r\left[1-x_{t}\right]-b y_{t}}, \\
y_{t+1}=x_{t}\left[1-\mathrm{e}^{-y_{t}}\right] .
\end{array}\right.
$$

We observe various types of dynamics having stable fixed point, chaotic bands, periodic windows for the non-dimensionalized model. We also observe the different types of bifurcations such as Neimark-Sacker, saddle-node and period-doubling bifurcation. We study the coexistence of the attractors of this non-dimensionalized model. Stable and oscillatory coexistence of host and parasitoid are observed for different values of parameters. We study the stable and unstable manifolds for different equilibrium points.

The case $P_{t}=0$, (Ricker dynamics)

Dynamics of this system for $P_{t}=0$ is the same as the dynamics which is given by the Ricker curve $N_{t+1}=N_{t} \mathrm{e}^{r\left(1-N_{t} / k\right)}$. The period-doubling bifurcations, periodic windows and chaos are observed in the Ricker curve [4] [5] [6] [7]. We present the Bifurcation diagram for Ricker map (Figure 1). Parameter $r$ is the reproductive rate of population and constant controlling the intrinsic changes in the population size.

In this case, $N=k$ is a steady state.

For Ricker map, we have,

$$
\begin{gathered}
f(N)=N \mathrm{e}^{r(1-N / k)}, \\
f^{\prime}(N)=\mathrm{e}^{r\left(1-N_{t} / k\right)}-\frac{N}{k} r \mathrm{e}^{r\left(1-N_{t} / k\right)} .
\end{gathered}
$$



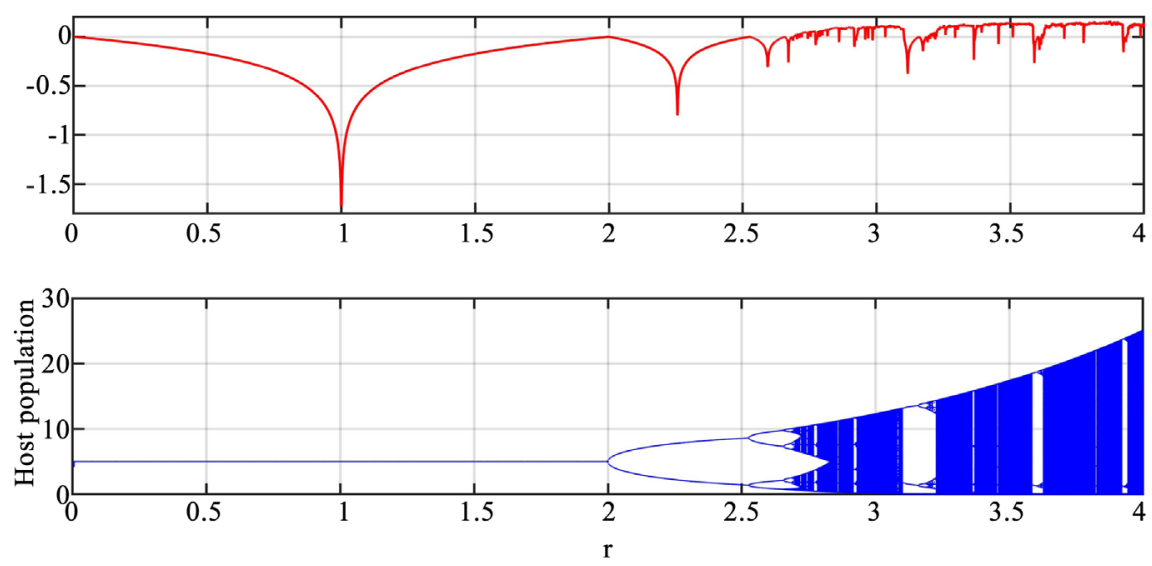

Figure 1. Bifurcation diagram and Lyapunov exponent for system (1) without parasitoid with $k=5$.

$N=k$, is a hyperbolic fixed point if $|1-r| \neq 1$ or $r \neq 0,2$ because $f^{\prime}(N)=1-r$ for $N=k$. For $r=2$, we have $f^{\prime}(N)=-1$ and in this case $N=k$ is a non hyperbolic fixed point.

For $0<r<2, N=k$ is asymptotically stable and for $r>2$, fixed point $N=k$ is unstable.

$$
\begin{gathered}
f^{\prime \prime}(N)=\frac{-2 r}{k} \mathrm{e}^{r\left(1-N_{t} / k\right)}+\frac{N r^{2}}{k^{2}} \mathrm{e}^{r\left(1-N_{t} / k\right)}, \\
f^{\prime \prime \prime}(N)=\frac{3 r^{2}}{k^{2}} \mathrm{e}^{r\left(1-N_{t} / k\right)}-\frac{N r^{3}}{k^{3}} \mathrm{e}^{r\left(1-N_{t} / k\right)} . \\
f^{\prime \prime \prime}(N=k)=\frac{3 r^{2}}{k^{2}}-\frac{r^{3}}{k^{2}} .
\end{gathered}
$$

If $f^{\prime}(N)=-1$, we use the following method [8].

Here for $N=k$ and $r=2$, we have $f^{\prime}(N)=-1$ and $S f(N)<0$. Therefore, $N=k$ is asymptotically stable because:

$$
S f(N)=\frac{\frac{4}{k^{2}}}{-1}=\frac{-4}{k^{2}}<0 .
$$

Therefore, for $r=2, N=k$ is asymptotically stable.

When $r$ will be more increased, the period-doubling bifurcation goes to chaotic bands. A stable coexistence is observed when $r<2$. The chaotic attractor appears when $r$ is approximately increased from 2.7. Afterward, complex dynamic patterns are observed.

In Figure 1, we see period-doubling bifurcation.

Furthermore, we see an attracting 3-cycle, which undergoes a sequence of period-doubling bifurcations. The period-3 windows are seen in bifurcation diagram. There are other windows visible in picture. Approximately, for $r>2.6$, system becomes deterministically chaotic, having as a strange attractor.

For $r>3.0$, extinction in this model is obviously. The complex dynamics of host population dynamics, including quasi-periodicity, period-doubling cascade, 
chaotic crisis, chaotic bands with narrow or wide periodic window, intermittent chaos, and supertransient behavior. In the absence of the parasitoid, host population density is regulated by intra-specific competition (Harper, 1977; Antonovics Levin, 1980; Watkinson, 1980), so we allow for density dependence in the host.

The case $b=a$, (Beddington, Free and Lawton model)

Case $b=a$ which is the density-dependent predator-prey model, is studied in [9]:

$$
N_{t+1}=N_{t} \mathrm{e}^{\left[r\left(1-N_{t} / k\right)-a P_{t}\right]}, \quad P_{t+1}=N_{t}\left(1-\mathrm{e}^{-a P_{t}}\right)
$$

The pair of difference equations were analyzed by Beddington, Free, and Lawton (1975).

The dynamics of this model are complicated. For some values of the parameters, this model has an asymptotically stable equilibrium with complex eigenvalues. When the parameter $a$ increases, this asymptotically stable equilibrium loses its stability and rise to an invariant closed curve. The dynamics of the invariant curve include periodic and quasi-periodic orbits. Eventually, the invariant curve loses its smoothness and breaks up into a chaotic attractor [9].

In model (2), we have one extinction fixed point $(0,0)$, one exclusion fixed point $(k, 0)$ and one coexistence fixed point.

The Jacobian matrix for system (2), has the following form:

$$
J=\left[\begin{array}{cc}
\frac{k-r N}{K} \mathrm{e}^{[r(1-N / k)-a P]} & -a N \mathrm{e}^{[r(1-N / k)-a P]} \\
1-\mathrm{e}^{-a P} & a N \mathrm{e}^{-a P}
\end{array}\right] .
$$

To analyze the local asymptotically stability, the Jacobian matrix at $(N, P)=(0,0)$ is calculated:

$$
J(0,0)=\left[\begin{array}{ll}
\mathrm{e}^{r} & 0 \\
0 & 0
\end{array}\right]
$$

Theorem 1. The extinction fixed point $(0,0)$ of system (2) is a saddle point and unstable.

Proof. The eigenvalues are $\lambda_{1,2}=\mathrm{e}^{r}, 0$. Since $r>0$, and hence $\mathrm{e}^{r}>1$, the eigenvalue $\mathrm{e}^{r}$ is greater than 1 that corresponds to a one-dimensional unstable manifold and $\lambda_{2}=0$ correspond to a one-dimensional stable manifold.

The Jacobian evaluated at the fixed point $(k, 0)$ is given by:

$$
J(k, 0)=\left[\begin{array}{cc}
1-r & -a k \\
0 & a k
\end{array}\right] .
$$

Theorem 2. The axial fixed point $(k, 0)$ of system (2) is asymptotically stable if we have, ak $<1,0<r<2$.

Proof. The eigenvalues of $J(k, 0)$ are $\lambda_{1}=1-r, \lambda_{2}=a k$. By linear stability theorem, $(k, 0)$ of system (2) is asymptotically stable if, $a k<1,0<r<2$.

Two issues remain unresolved. They are when $\lambda_{1}=1-r=-1$ and $k a=1$.

Here, we apply the center manifold theorem. 
Jordan form for the case $a k=1$ at $(k, 0)$ is given by,

$$
D f(x, y)=\left[\begin{array}{cc}
1-r & -a k \\
0 & 1
\end{array}\right] \text {. }
$$

We make a change of variables. Let $x=u+k$ and $y=v$. With this change of variables, $(k, 0)$ coincide with origin and system (2) by the following form,

$$
\left\{\begin{array}{l}
u_{t+1}=k a u_{t}+f_{1}\left(u_{t}, v_{t}\right), \\
v_{t+1}=(1-r) v_{t}+g_{1}\left(u_{t}, v_{t}\right) .
\end{array}\right.
$$

The non-linear terms for above system are the following form,

$$
\left\{\begin{array}{l}
f_{1}\left(u_{t}, v_{t}\right)=\left(u_{t}+k\right) \mathrm{e}^{r\left(1-\left(u_{t}+k\right) / k\right)}-k a u_{t}-k, \\
g_{1}\left(u_{t}, v_{t}\right)=-\left(u_{t}+k\right)\left(-1+\mathrm{e}^{\left(-v_{t} / k\right)}\right)-(1-r) v_{t} .
\end{array}\right.
$$

Let us assume that the map $h_{1}$ takes the form

$$
v=h_{1}(u)=\alpha u^{2}+\beta u^{3}+O\left(u^{4}\right), \quad \alpha, \beta \in \mathbb{R}
$$

Now we are going to compute the constants $\alpha$ and $\beta$. The function $h$ must satisfy the center manifold equation

$$
N h_{1}(u)=h_{1}(B u)+g_{1}\left(u, h_{1}(u)\right)-A h_{1}(u)-f_{1}\left(u, h_{1}(u)\right)=0
$$

Without considering the exponential terms, we have the following equation,

$$
k_{2} u^{2}+k_{3} u^{3}=0 .
$$

Solving the system $k_{2}=0$ and $k_{3}=0$ yields the unique solution $\alpha=0$ and $\beta=0$.

Thus on the center manifold $v=0$, we have the following map

$$
L(u)=(u+k) \mathrm{e}^{r\left(1-\frac{u+k}{k}\right)}-k .
$$

For $a k=1,(u, v)=(0,0)$, we have $L^{\prime}(0)=1-r,(u, v)=(0,0)$ is asymptotically stable, if $|1-r|<1$ and it is unstable, if $|1-r|>1$.

The second case is $1-r=-1$. Jordan form for the case $1-r=-1$ at $(k, 0)$ is given by,

$$
D f(x, y)=\left[\begin{array}{cc}
-1 & -a k \\
0 & a k
\end{array}\right]
$$

With this change of variables $x=u+k$ and $y=v$ system (2) for $1-r=-1$ by the following form,

$$
\left\{\begin{array}{l}
u_{t+1}=k a u_{t}+f_{2}\left(u_{t}, v_{t}\right), \\
v_{t+1}=-v_{t}+g_{2}\left(u_{t}, v_{t}\right) .
\end{array}\right.
$$

The non-linear terms for above system are the following form,

$$
\left\{\begin{array}{l}
f_{2}\left(u_{t}, v_{t}\right)=\left(u_{t}+k\right) \mathrm{e}^{r\left(1-\left(u_{t}+k\right) / k\right)}+k a u_{t}-k, \\
g_{2}\left(u_{t}, v_{t}\right)=-\left(u_{t}+k\right)\left(-1+\mathrm{e}^{\left(-a v_{t}\right)}\right)+v_{t} .
\end{array}\right.
$$

Let us assume that the map $h_{2}$ takes the form 


$$
u=h_{2}(v)=\delta v^{2}+\gamma v^{3}+O\left(v^{4}\right), \quad \alpha, \beta \in \mathbb{R}
$$

Now we are going to compute the constants $\delta$ and $\gamma$. The function $h_{2}$ must satisfy the center manifold equation

$$
N h_{2}(v)=h_{2}(A v)+f_{2}\left(v, h_{2}(v)\right)-B h_{2}(v)-g_{2}\left(v, h_{2}(v)\right)=0
$$

Solving this equation, we have the unique solution $\delta=0$ and $\gamma=0$. Thus on the center manifold $u=0$, we have the following map

$$
Q(v)=k\left(1-\mathrm{e}^{-a v}\right) .
$$

For $1-r=-1$, we have $Q^{\prime}(0)=a k .(u, v)=(0,0)$ is asymptotically stable, if $|a k|<1$ and it is unstable, if $|a k|>1$.

The coexistence equilibrium for system (2) is

$$
N^{*}=\frac{k\left(r-a P^{*}\right)}{r},
$$

and $P^{*}$ is the positive root of the equation,

$$
P^{*}=\frac{k\left(r-a P^{*}\right)}{r}\left(1-\exp \left(-a P^{*}\right)\right) .
$$

The Jacobian at the coexistence equilibrium point can be simplified at the form:

$$
J\left(N^{*}, P^{*}\right)=\left[\begin{array}{cc}
1+r+a P^{*} & -a N^{*} \\
\frac{P^{*}}{N^{*}} & a\left(N^{*}-P^{*}\right)
\end{array}\right] .
$$

For the coexistence equilibrium point we have,

$$
\begin{aligned}
& \operatorname{tr}\left(J\left(N^{*}, P^{*}\right)\right)=1-r+a P^{*}+a N^{*}-a P^{*}, \\
& \operatorname{det}\left(J\left(N^{*}, P^{*}\right)\right)=\left(1-r+a P^{*}\right)\left(a N^{*}-a P^{*}\right)+a P^{*} .
\end{aligned}
$$

Theorem 3. The coexistence equilibrium point in system (2) is asymptotically stable if we have:

$$
\left|1-r+a P^{*}+a N^{*}-a P^{*}\right|<1+\left(1-r+a P^{*}\right)\left(a N^{*}-a P^{*}\right)+a P^{*}<2 .
$$

Proof. The proof is straightforward using the fact that the positive fixed point of system is asymptotically stable if and only if

$$
|\operatorname{tr}(J)|<1+\operatorname{det}(J)<2
$$

The above inequality is the sufficient condition for the local stability of the fixed point and necessary condition for the roots of the characteristic equation to be inside the complex plane.

In Figure 2, we choose $a=0.2$, initial host density $N=11$, initial parasitoid density $P=1$ and we plot phase portraits for system (2). We see for $r>0.5$ (with considering the bifurcation diagram for $k=14.47$ ), the equilibrium is asymptotically stable.

For the case $a=b$, Taylor expansion of plant equation for $r=2$ on the invariant manifold $P_{t}=0$ has the condition of the period-doubling bifurcation correspond to Ricker dynamics. 

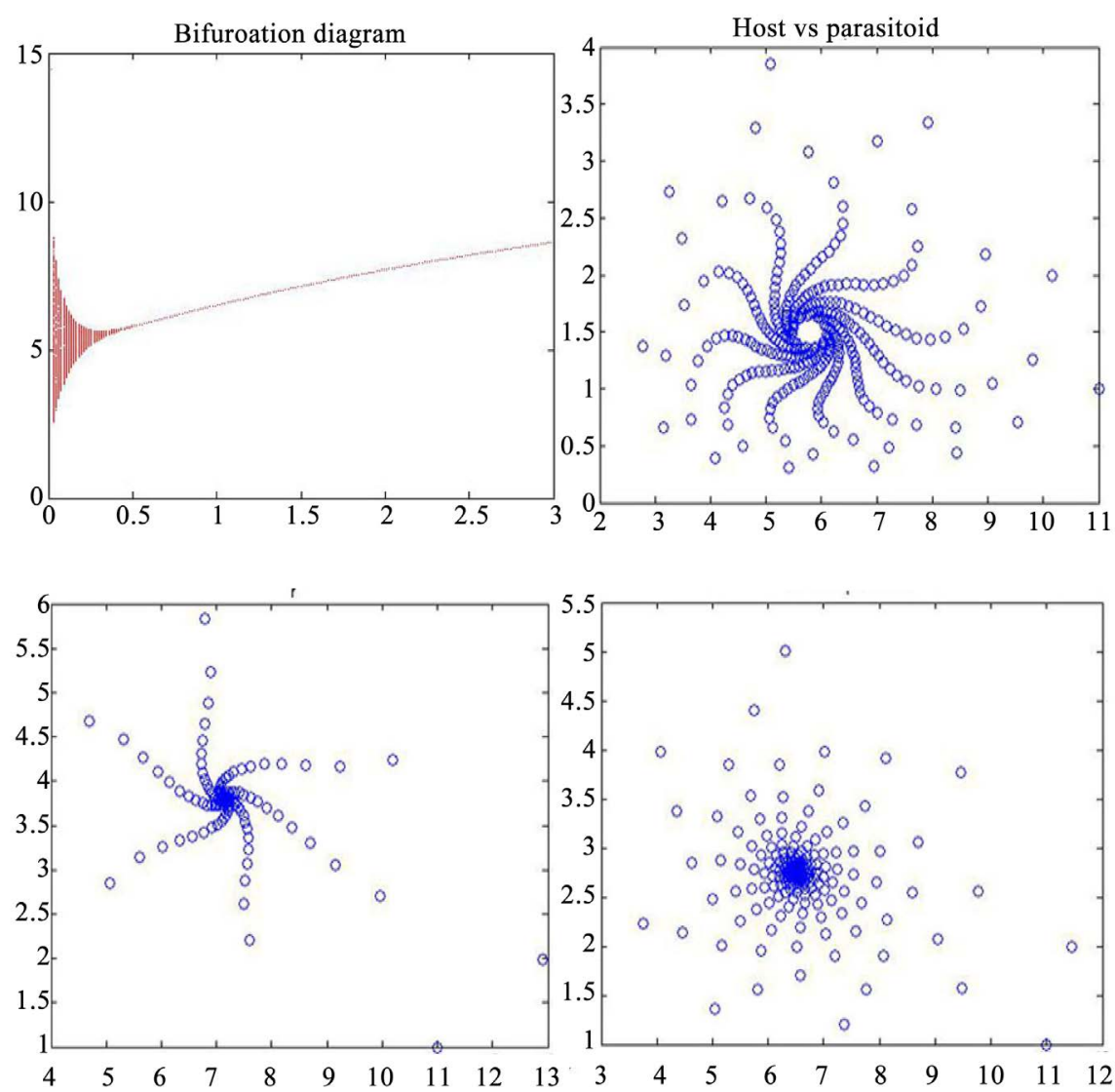

Figure 2. Bifurcation diagram for system (2) with $k=14.47, a=0.2$ (up-left). Phase portraits of the system (2) for $r=0.5$ of taking $k=14.47, a=0.2$ (up-right). Phase portraits of the system (2) for $r=1$ (down-right). Phase portraits of the system (2) for $r=1.5$ (down-left).

\section{The Case $b \neq a$}

In this section, we investigate rescaling (nondimensionalization) host-parasitoid dynamics (1) with considering $b \neq a$.

The bifurcation diagram for system (1) is displayed in Figure 3, shows for $r=2.5$, the Neimark-Sacker bifurcation occurs in system (1).

The phase portraits of system (1) are displayed in Figure 4, shows for $r \approx 1.4, a=40, b=1$ and initial values $N_{0}=1.09=P_{0}$, the Neimark-Sacker bifurcation occurs in system (1). For $a=40$, when the parameter $r$ is changed, the asymptotically stable fixed point loses its stability and to be an attracting closed invariant curve gradually. When the values of $r$ increase, radius of the curve enhances.

We draw the phase portrait for different values of $r$ (see Figure 5).

To nondimensionalized system (1), Replace each of variables with a quantity scaled relative to a characteristic unit of measure to be determined. By rescaling $N$ and $P$, one of the parameters can be set to be 1 without loss of generality (e.g. set $a=1$ ), consequently, we have a two parameters non-dimensionalized system: 


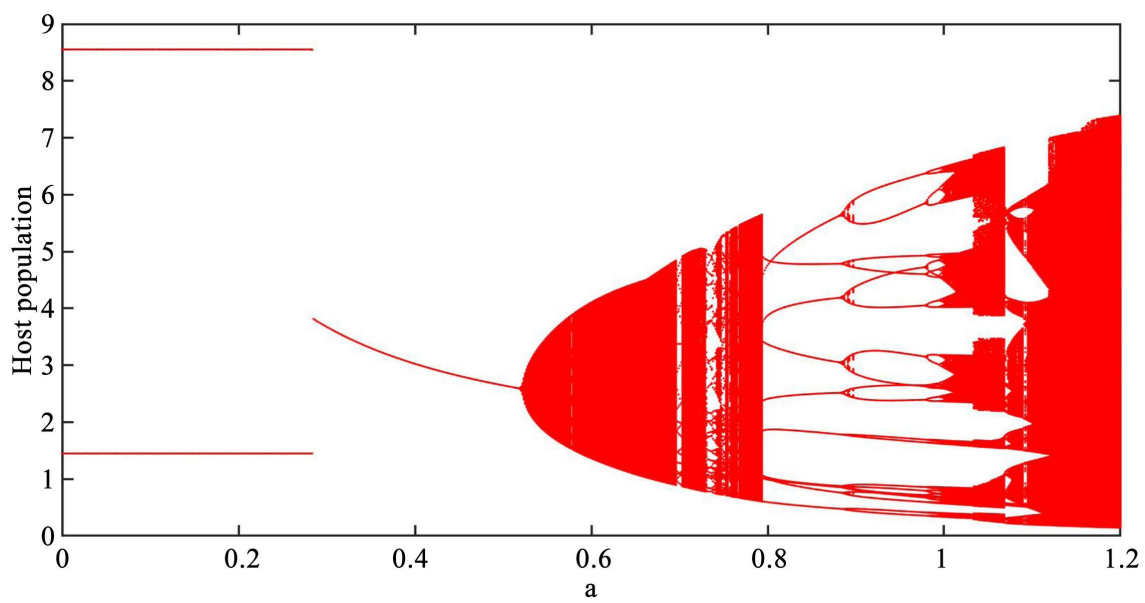

Figure 3. Bifurcation diagram for Host population in system (1) initial value $k=5$.
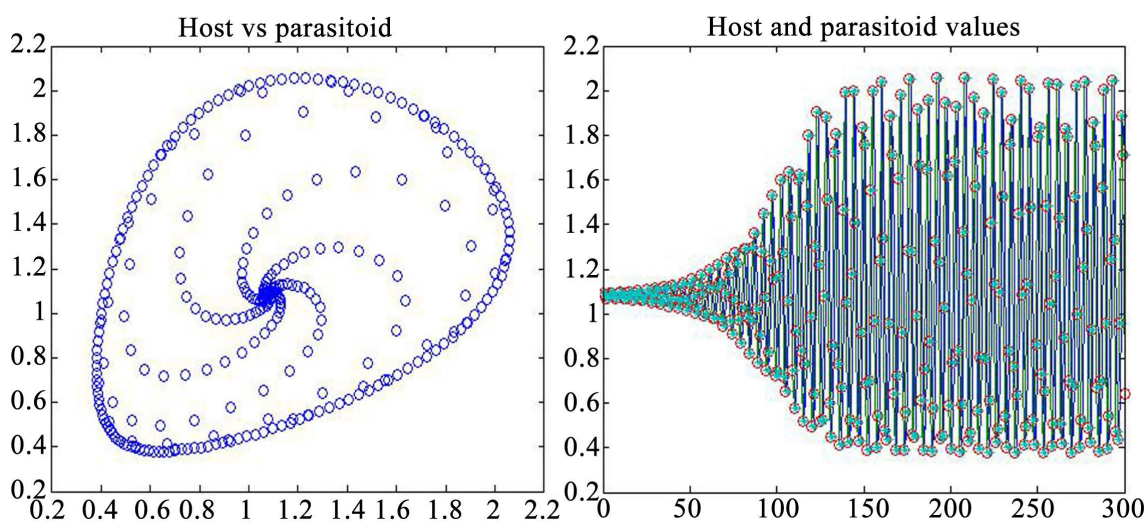

Figure 4. The phase portraits of the system (1) with $a=40, b=1$ and initial value $k=5$ (left), Time series (right). An invariant closed circle bifurcates from equilibrium.

$$
\left\{\begin{array}{l}
x_{t+1}=x_{t} \mathrm{e}^{r\left[1-x_{t}\right]-b y_{t}}, \\
y_{t+1}=x_{t}\left[1-\mathrm{e}^{-y_{t}}\right] .
\end{array}\right.
$$

The equilibrium solutions and the local asymptotic stability of the model (3) are analyzed.

We study the different types of bifurcation such as Neimark-Sacker, saddle-node and period-doubling bifurcation.

\subsection{Solutions and Local Asymptotic Stability}

In this subsection, we study the equilibrium solutions and the local asymptotic stability of the model (3).

To analysis the local asymptotically stability, Jacobian matrix is calculated and evaluated at equilibrium.

In model (3), we have one extinction fixed point $(0,0)$, one exclusion fixed point $(1,0)$ and one coexistence fixed point.

The fixed point $\left(x_{t}, y_{t}\right)=(0,0)$ represents the extinction of both species. If both of the populations are at 0 , they will continue to be so indefinitely. 

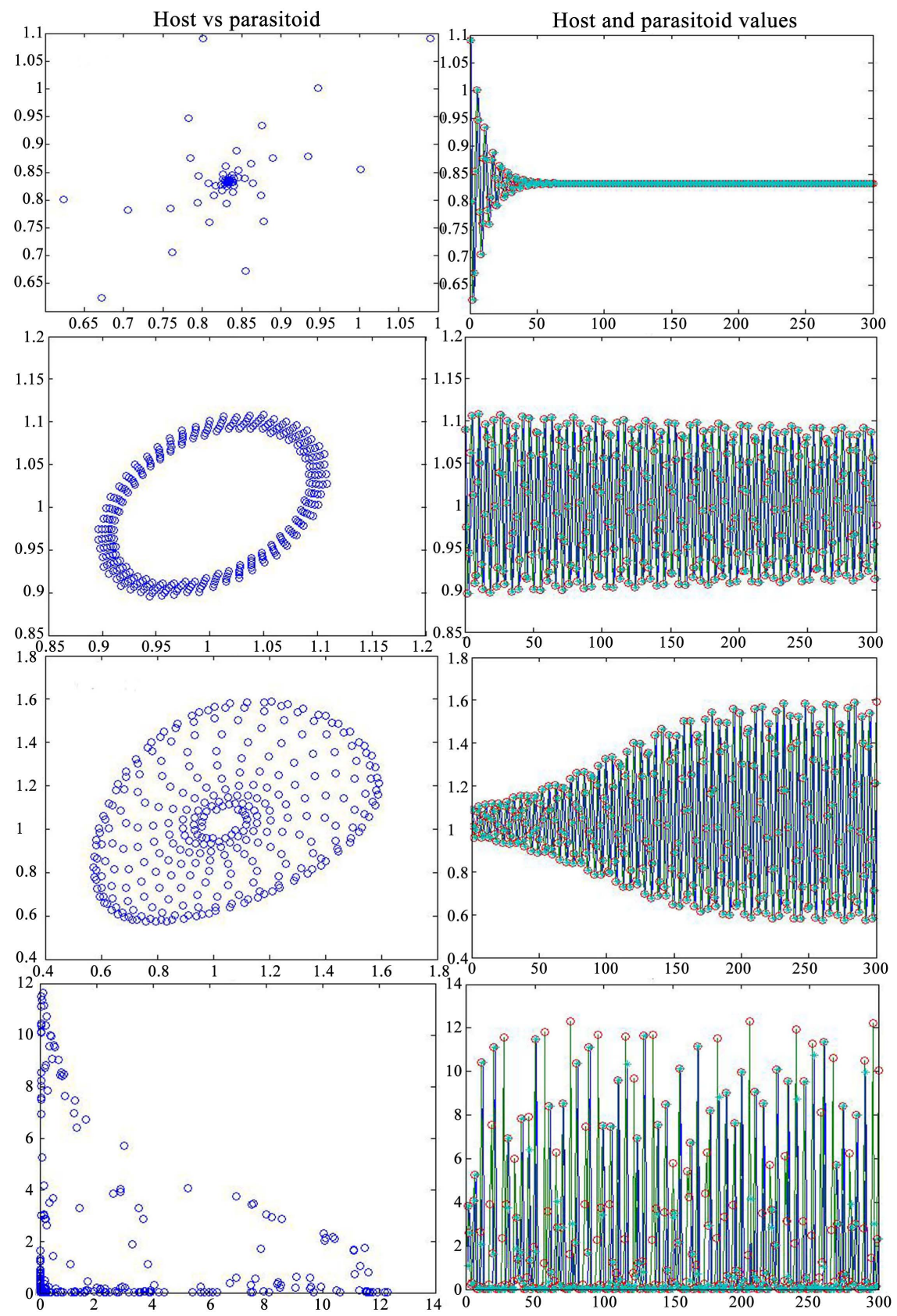

Figure 5. The phase portraits of the system (1) with $a=40, b=1$ and initial value $k=5$, from up $r=1, r=1.25, r=1.3, r=3$. This important bifurcation gives rise to sustained stable oscillations of the host and parasitoid populations; the amplitude of the oscillations grows with changing $r$.

The Jacobian matrix for system (3), has the following form:

$$
J=\left[\begin{array}{cc}
\left(1-r x_{t}\right) \mathrm{e}^{\left[r\left(1-x_{t}\right)-y_{t}\right]} & -b x_{t} \mathrm{e}^{\left[r\left(1-x_{t}\right)-y_{t}\right]} \\
1-\mathrm{e}^{-y_{t}} & x_{t} \mathrm{e}^{-y_{t}}
\end{array}\right] .
$$

One equilibria is the zero equilibrium, where $\bar{x}=0$ and $\bar{y}=0$.

To analyze the local asymptotically stability, the Jacobian matrix at $(x, y)=(0,0)$ 
is calculated:

$$
J(0,0)=\left[\begin{array}{cc}
\mathrm{e}^{r} & 0 \\
0 & 0
\end{array}\right] .
$$

The characteristic polynomial for $J(0,0)$ is the following form,

$$
P_{0}(\lambda)=\lambda^{2}-\mathrm{e}^{r} \lambda \text {. }
$$

Note. If $\lambda_{i}$ are eigenvalues for Jacobian matrix at the equilibrium point and we have $\left|\lambda_{i}\right|<1$, then the equilibrium is locally asymptotically stable.

The eigenvalues are $\lambda_{1,2}=\mathrm{e}^{r}, 0$. Since $r>0$, and hence $\mathrm{e}^{r}>1$, the eigenvalue $\mathrm{e}^{r}$ is greater than 1 that corresponds to a one-dimensional unstable manifold and $\lambda_{2}=0$ correspond to a one-dimensional stable manifold.

Theorem 4. The extinction fixed point $(0,0)$ of the system (3) is a saddle point and unstable.

Proof. The eigenvalues for Jacobian matrix $J(0,0)$ are $\lambda_{1,2}=\mathrm{e}^{r}, 0$. Since $r>0,\left|\mathrm{e}^{r}\right|>1$ and according to Linearised Stability Theorem [10], $(0,0)$ is unstable.

According to part (e),

$$
s^{2}+4 t=\mathrm{e}^{2 r}>0 \quad \text { and } \quad\left|\mathrm{e}^{r}\right|>|1-0|=1 .
$$

Consequently, $(0,0)$ is a saddle point.

The Jacobian evaluated at the fixed point $(1,0)$ is given by (Axial fixed point $(1,0)$ in the absence of parasitoid):

$$
J(1,0)=\left[\begin{array}{cc}
1-r & -b \\
0 & 1
\end{array}\right] .
$$

The characteristic polynomial for $J(1,0)$ is the following form,

$$
P_{0}(\lambda)=\lambda^{2}-(2-r) \lambda+(1-r) .
$$

The eigenvalues of $J(1,0)$ are $\lambda_{1}=1-r, \lambda_{2}=1$.

Theorem 5. The axial fixed point $(1,0)$ of system (3) is asymptotically stable if we have, $0<r<2$.

Proof. It is straightforward using Linearised Stability Theorem [10] and or Jury condition [11].

When $r=2$, we have the most degenerate case with eigenvalues $\lambda_{1}=-1$, $\lambda_{2}=1$.

Taylor expansion of plant equation for $r=2$ on the invariant manifold $y=0$ for system (3) gives

$$
u_{t+1}=-u_{t}+\frac{2 u_{t}^{3}}{3}-\frac{2 u_{t}^{4}}{3}+\frac{2 u_{t}^{5}}{5}+O\left(u_{t}^{6}\right) .
$$

This equation has the condition of occurrence the period-doubling bifurcation correspond to Ricker dynamics.

The coexistence equilibrium for system (3) is

$$
x^{*}=\frac{r-b y^{*}}{r} \text { and } y^{*}=x^{*}\left(1-\mathrm{e}^{-y^{*}}\right) .
$$


Let $\left(x^{*}, y^{*}\right)$ be an coexistence equilibrium of model (3). Then the coexistence equilibria are the intersection points of bellow functions in the first quadrant $Q^{+}$.

$$
f_{1}(y)=\frac{r-b y}{r}, \quad f_{2}(y)=\frac{y}{1-\mathrm{e}^{-y}} .
$$

Clearly, $f_{1}(y)$ is a decreasing linear function. It is easy to show that $f_{2}(y)$ is increasing.

The Jacobian at the coexistence equilibrium point can be simplify at the form:

$$
J\left(x^{*}, y^{*}\right)=\left[\begin{array}{cc}
1-r x^{*} & -b x^{*} \\
\frac{y^{*}}{x^{*}} & x^{*}-y^{*}
\end{array}\right] .
$$

For the coexistence equilibrium point we have,

$$
\begin{aligned}
& \operatorname{tr}\left(J\left(x^{*}, y^{*}\right)\right)=1-r x^{*}+x^{*}-y^{*}, \\
& \operatorname{det}\left(J\left(x^{*}, y^{*}\right)\right)=\left(1-r x^{*}\right)\left(x^{*}-y^{*}\right)+b y^{*} .
\end{aligned}
$$

It will be shown the coexistence equilibrium point is asymptotically stable if the following Theorem is satisfied:

Theorem 6. The coexistence equilibrium point in system (3) is asymptotically stable if we have:

$$
\left|1-r x^{*}+x^{*}-y^{*}\right|-1<\left(1-r x^{*}\right)\left(x^{*}-y^{*}\right)+b y^{*}<1
$$

Proof. This is straightforward using local stability theorems in [10] [11] [12].

The host-parasitoid interaction exhibit simple steady state dynamics but it is not clear what biological mechanisms ensure that the inequalities in this equation holds, since the dynamical outcomes are very sensitive to two parameters $b$ and $r$ (Mukherjee and Das and Kesh).

Theorem 7. For coexistence equilibrium point of system (3) we have a stable and an unstable manifolds, when one of the following conditions are satisfied [8]:

1) $\left(1-r x^{*}\right)\left(x^{*}-y^{*}\right)+b y^{*}<-r x^{*}+x^{*}-y^{*}$, $\left(1-r x^{*}\right)\left(x^{*}-y^{*}\right)+b y^{*}>-2+r x^{*}-x^{*}+y^{*}$,

2) $\left(1-r x^{*}\right)\left(x^{*}-y^{*}\right)+b y^{*}>-r x^{*}+x^{*}-y^{*}$, $\left(1-r x^{*}\right)\left(x^{*}-y^{*}\right)+b y^{*}<-2+r x^{*}-x^{*}+y^{*}$,

3) $\left(1-r x^{*}\right)\left(x^{*}-y^{*}\right)+b y^{*}<\frac{\left(1-r x^{*}+x^{*}-y^{*}\right)^{2}}{4}$, $\left(1-r x^{*}\right)\left(x^{*}-y^{*}\right)+b y^{*}>1$, $\left(1-r x^{*}\right)\left(x^{*}-y^{*}\right)+b y^{*}>-2+r x^{*}-x^{*}+y^{*}$

4) $\left(1-r x^{*}\right)\left(x^{*}-y^{*}\right)+b y^{*}<\frac{\left(1-r x^{*}+x^{*}-y^{*}\right)^{2}}{4}$, $\left(1-r x^{*}\right)\left(x^{*}-y^{*}\right)+b y^{*}>-r x^{*}+x^{*}-y^{*}$, $\left(1-r x^{*}\right)\left(x^{*}-y^{*}\right)+b y^{*}>1$

Proof. It is straightforward using Linearised Stability Theorem [10]. 
Host-parasitoid models with Ricker dynamics (hump dynamics) in host have much complicated dynamics [7].

\subsection{Bifurcations}

In this section, we analyse the bifurcations of extinction fixed point $(0,0)$, exclusion fixed point $(1,0)$. Here we use the Theorems from Elaydi [8].

Remark 1. The saddle-node bifurcation occurs when the Jacobian matrix has an eigenvalue equal to 1 . This is equivalent to saying that we cross the line $\operatorname{det}\left(J^{*}\right)=\operatorname{tr}\left(J^{*}\right)-1$ from the stability region [4]. The saddle-node bifurcation is the type I intermittency ${ }^{1}$ (route to chaos). Hilborn has discussed about types of intermittency in his book [13].

Remark 2. The period-doubling bifurcation occurs when the Jacobian matrix has an eigenvalue equal to -1 . In the T-D plane this occurs as we cross the line $\operatorname{det}\left(J^{*}\right)=-\operatorname{tr}\left(J^{*}\right)-1$ from the stability region [8].

Result. For the axial fixed point $(1,0), \lambda_{2}=1-r=-1$, This leads $r=2$. In this case the period-doubling bifurcation occurs.

Remark 3. When $\operatorname{det}\left(J^{*}\right)=1$ and $\left|\operatorname{tr}\left(J^{*}\right)\right|<2$, Neimark-Sacker bifurcation occurs [8].

Theorem 8 We have a Neimark-Sacker bifurcation at the coexistence equilibrium point for system (3) when we have:

$$
1-r x^{*}+x^{*}-y^{*}=1, \quad-2<\left(1-r x^{*}\right)\left(x^{*}-y^{*}\right)+b y^{*}<2 .
$$

Proof. Using Remark 3:

$$
\begin{gathered}
\operatorname{det} J^{*}=1 \Rightarrow 1-r x^{*}+x^{*}-y^{*}=1, \\
-2<\operatorname{tr}\left(J^{*}\right)<2 \Rightarrow-2<\left(1-r x^{*}\right)\left(x^{*}-y^{*}\right)+b y^{*}<2 .
\end{gathered}
$$

Here we give the necessary conditions for Neimark-Sacker bifurcations in our two models.

\subsection{Non-Unique Dynamics and Bistability}

It is possible that in a discrete-time dynamical system, different types of attractors such as equilibrium point vs. chaotic attractors, periodic vs. quasiperiodic attractors and quasiperiodic vs. chaotic attractors coexist. This phenomenon is called non-unique dynamics and coexistence of attractors which implies that in the bifurcation diagrams, routes to chaos through period doubling bifurcations, is dependent on initial conditions and therefore non-unique [14] [15] [16]. Non-uniqueness induces a problem for predicting and controlling the dynamics in different of engineering and biological problems. The complex dynamics in a host-parasitoid model induce a multiple attractor.

We have bistability between two attractors, an interior one and a boundary one. Bistable dynamics is often generated a threshold level below which the parasitoid will die out regardless of the host population levels (Kang; Aarmbruster; Kuang, 2008) [17]. Complex dynamics in a host-parasitoid model can lead to a ${ }^{1}$ In intermittency, the behaviour of system switch between periodic, quasi periodic and chaotic. 
multiple attractor case. The bistable system can switch from one stable mode to the other. We have stable interior attractors (equilibria, periodic orbits and strange attractors) and stable attractors in the $\mathrm{x}$-boundary Ricker dynamics corresponding to the $y_{t}=0$ and the extinction of parasitoid (periodic orbits and strange attractors). The stability of $\left\{x_{i}\right\}_{t=1}^{t=N}$ on the invariant manifold $y=0$ depends on the eigenvalues of the following Jacobian matrix,

$$
J=\prod_{t=1}^{t=N}\left(\begin{array}{cc}
\mathrm{e}^{r\left(1-x_{t}\right)}\left(1-r x_{t}\right) & -b x_{t} \mathrm{e}^{r\left(1-x_{t}\right)} \\
0 & x_{t}
\end{array}\right) .
$$

Then the eigenvalues of $J$ are,

$$
\prod_{t=1}^{t=N} \mathrm{e}^{r\left(1-x_{t}\right)}\left(1-r x_{t}\right), \quad \prod_{t=1}^{t=N} x_{t} .
$$

Since $\left\{x_{i}\right\}_{t=1}^{t=N}$ is a stable periodic orbit of the Ricker model, i.e., the so-called internal stability (Kon 2006) [15],

$$
\left|\prod_{t=1}^{t=N} \mathrm{e}^{r\left(1-x_{t}\right)}\left(1-r x_{t}\right)\right|<1
$$

Hence if the following inequality holds, $\left\{x_{i}\right\}_{t=1}^{t=N}$ is stable, that is system (3) is not permanent (Kon 2001) [16]:

$$
\left(\prod_{t=1}^{t=N} x_{t}\right)^{\frac{1}{N}}<1
$$

If $\left|\lambda_{1,2}\right|<1$, this is the case of bistability.

In Figure 6, for different values of $r$, we see that two alternative attractors coexist. This figure shows the existence of quasi-periodic attractors, circular curves enclosing fixed point and chaotic attractors for different values of $r$. As $r$ is changing periodic behavior is observed and replaced with the chaotic bands in some intervals of parameter values. The chaotic bands again disappear and form a periodic behavior. The regular and chaotic behaviors are alternating as we increase $r$.

\section{Conclusion}

In this paper, we studied a discrete-time host-parasitoid model which is non-dimensionalized Nicholson-Bailey model. The model shows rich features

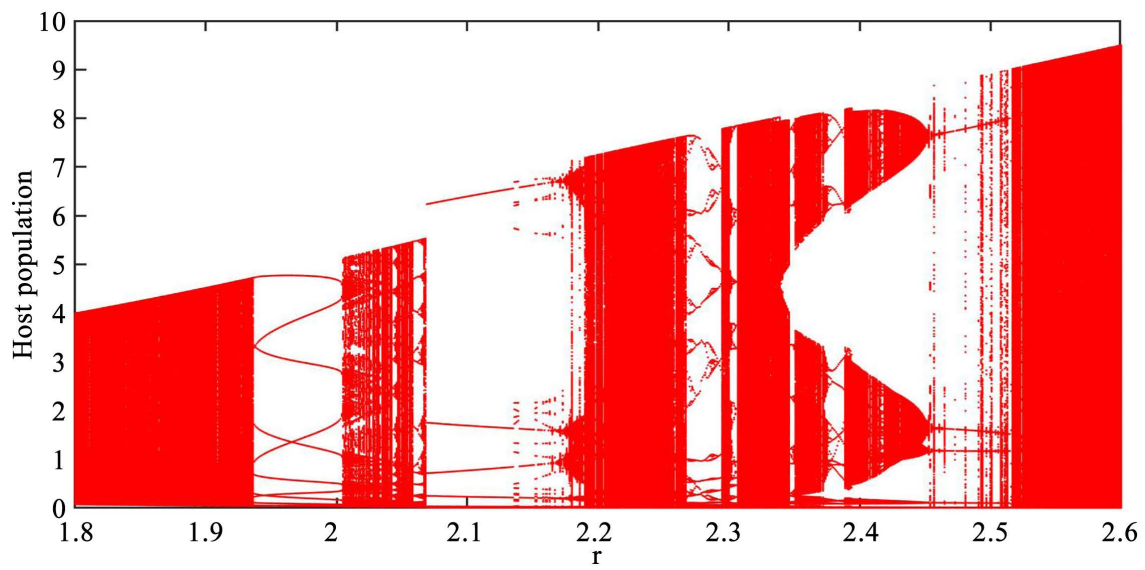

Figure 6. Coexistence of attractors with different values of $r$. 
and more complicated dynamics compared to the general Nicholson-Bailey model. The equilibrium solutions have been calculated and their local asymptotic stability has been analyzed. This model demonstrates different types of behaviors from regular and stable behavior and periodic windows to irregular dynamics and chaos. Bifurcation analysis has been done with respect to intrinsic growth rate $r$ and search efficiency $a$. Transition routes to chaos were established via period-doubling bifurcation. The necessary conditions of occurrence of the period-doubling, Neimark-Sacker and saddle-node bifurcations have been obtained. Type I intermittency (Neimark-Sacker bifurcation) and type II intermittency (saddle-node bifurcation) were observed. We presented the conditions under which the general two-dimensional map has a stable and an unstable manifold for different equilibrium points. For $P_{t}=0$, this system was similar to the dynamics of Ricker map. For $b=a$, this system was studied by Beddington and Free and Lawton. As we change $r$ and $a$, the dynamics on the invariant curve exhibited periodic and quasi-periodic orbits. Furthermore, with further increasing the parameter values, we noticed that the invariant closed curves lost their stability and broke up into chaotic attractors. We observed various types of non-unique dynamics and coexistence of different attractors such as stable fixed point, chaotic bands, periodic windows, quasi-periodic orbits. We also observed attractor crises. The present work helped us to understand the dynamical behavior of host-parasitoid interactions with intraspecific which can be used to improve the classical biological control of parasitiods.

\section{Conflicts of Interest}

The author declares no conflicts of interest regarding the publication of this paper.

\section{References}

[1] Zhao, M., Zhang, L. and Zhu, J. (2011) Dynamics of a Host-Parasitoid Model with Prolonged Diapause for Parasitoid. Communications in Nonlinear Science and Numerical Simulation, 16, 455-462. https://doi.org/10.1016/j.cnsns.2010.03.011

[2] Flores, J.D. (2011) Mathematical Modeling Chapter III Nicholson-Bailey Model. $\mathrm{PhD}$.

[3] Hone, A.N.W., Irle, M.V. and Thurura, G.W. (2010) On the Neimark-Sacker Bifurcation in a Discrete Predator-Prey System. Journal of Biological Dynamics, 4, 594-606. https://doi.org/10.1080/17513750903528192

[4] Azizi, T. and Kerr, G. (2020) Synchronized Cycles of Generalized Nicholson-Bailey Model. American Journal of Computational Mathematics, 10, 147-166. https://doi.org/10.4236/ajcm.2020.101009

[5] Ricker, W.E. (1954) Stack and Recruitment. Journal of the Fisheries Research Board of Canada, 11, 559-623. https://doi.org/10.1139/f54-039

[6] Azizi, T. and Kerr, G. (2020) Chaos Synchronization in Discrete-Time Dynamical Systems with Application in Population Dynamics. Journal of Applied Mathematics and Physics, 8, 406-423. https://doi.org/10.4236/jamp.2020.83031

[7] Azizi, T. (2015) Dynamics of a Discrete-Time Plant-Herbivore Model. Caspian 
Journal of Mathematical Sciences, 4, 241-256.

[8] Elaydi, S. (2008) Discrete Chaos: With Applications in Science and Engineering. 2nd Edition, Chapman and Hall/CRC, Boca Raton, London, New York.

[9] Beddington, J.R., Free, C.A. and Lawton, J.H. (1975) Dynamic Complexity in Predator-Prey Models Framed in Difference Equations. Nature, 255, 58-60. https://doi.org/10.1038/255058a0

[10] Burgic, D., Kalabusic, S. and Kulenovic, M.R.S. (2008) Non Hyperbolic Dynamics for Competitive Systems in the Plane and Global Period-Doubling Bifurcations. Advances in Dynamical Systems and Applications, 3, 229-249.

[11] Murray, J.D. (1993) Mathematical Biology. Springer-Verlag, New York. https://doi.org/10.1007/978-3-662-08542-4

[12] Liu, X. and Xiao, D. (2007) Complex Dynamic Behaviour of a Discrete Time Predator-Prey System. Chaos, Solitons and Fractals, 32, 80-94.

https://doi.org/10.1016/j.chaos.2005.10.081

[13] Hilborn, R.C. (2000) Chaos and Nonlinear Dynamics. 2nd Edition, Oxford University Press, New York. https://doi.org/10.1093/acprof:oso/9780198507239.001.0001

[14] Kaitala, V., Ylikarjula, J. and Heino, M. (2000) Non-Unique Population Dynamics: Basic Patterns. Ecological Modelling, 135, 127-134.

https://doi.org/10.1016/S0304-3800(00)00357-4

[15] Kon, R. (2006) Multiple Attractors in Host-Parasitoid Interactions: Coexistence and Extinction. Mathematical Biosciences, 201, 1383-1394. https://doi.org/10.1016/j.mbs.2005.12.010

[16] Kon, R. and Takeuchi, Y. (2001) Permanence of Host-Parasitoid Systems. Nonlinear Analysis-Theory Methods and Applications, 47, 89-101.

https://doi.org/10.1016/S0362-546X(01)00273-5

[17] Yun, K., Armbruster, D. and Yang, K. (2008) Dynamics of a Plant-Herbivore Model. Journal of Biological Dynamics, 2, 89-101.

https://doi.org/10.1080/17513750801956313 